\title{
NEEF: A NOVEL ENERGY EFFICIENT FUZZY LOGIC BASED CLUSTERING PROTOCOL FOR WIRELESS SENSOR NETWORK
}

\author{
ANSHU KUMAR DWIVEDI*AND A.K. SHARMA
}

\begin{abstract}
The uttermost requirement of the wireless sensor network is prolonged lifetime. Unequal energy degeneration in clustered sensor nodes lead to the premature death of sensor nodes resulting in a lessened lifetime. Most of the proposed protocols primarily choose cluster head on the basis of a random number, which is somewhat discriminating as some nodes which are eligible candidates for cluster head role may be skipped because of this randomness. To rule out this issue, we propose a deterministic novel energy efficient fuzzy logic based clustering protocol (NEEF) which considers primary and secondary factors in fuzzy logic system while selecting cluster heads. After selection of cluster heads, non-cluster head nodes use fuzzy logic for prudent selection of their cluster head for cluster formation. NEEF is simulated and compared with two recent state of the art protocols, namely SCHFTL and DFCR under two scenarios. Simulation results unveil better performance by balancing the load and improvement in terms of stability period, packets forwarded to the base station, improved average energy and extended lifetime.
\end{abstract}

Key words: Energy Efficiency, Wireless Sensor Network, Clustering, Fuzzy Logic, Cluster Head

AMS subject classifications. 68M11, 94D05

1. Introduction. In recent past decades, wireless sensor network (WSN) has emerged as a vital part of our daily life. With the drastic progression of microelectronics technology which consumes low power in electronic circuitry, WSN is applied in diverse real-time applications like commercial monitoring, healthcare sensing, surrounding monitoring, battlefield surveillance etc. [1]. WSN contains sensor nodes (SN) which can experience, accumulate and compute information from the environment and also maintain it for a protracted time frame. A WSN is a blend of four subsystems altogether with a sensing module, a transceiver module, a processing module, and a power supply module (battery) [2]. These SNs use battery for energy delivery, limited memory for collecting information from the vicinity in which these are deployed and also microprocessor for processing the data and later transferring it to the base station (BS). So, it has been a premier issue for researchers to devise a mechanism to use the energy of SN effectively. WSN can be classified as heterogeneous or homogeneous networks [3]. In a homogeneous network, all nodes possess equal capacities in terms of processing, memory, radio range and energy, whereas in a heterogeneous network, it may be different. Clustering is one of the better solutions for sparing power of SNs and extending network lifetime [4]. Clustering is a method wherein all the SNs are grouped according to some criteria and each group is headed by one of the nodes called a cluster head $(\mathrm{CH})$ [5]. The $\mathrm{CH}$ compresses the data supplied by their cluster members (CMs) via statistics fusion to reduce the redundancy and improving the power dissipation rate of the network.

Various clustering algorithms were proposed in the last decades like LEACH [4], PEGASIS [6], HEED [7], SEP [8], LEASE [9], EDFCM [10], SPEZ [11] etc. In WSN, there are two types of information gathering schemes: Hierarchical and Non-Hierarchical. In a hierarchical scheme, SNs communicate the records to the $\mathrm{BS}$ via $\mathrm{CH}$ in one hop while, in a non-hierarchical scheme, SNs send the records in single and/or multi-hop to the $\mathrm{BS}$ via a $\mathrm{CH}$ resulting in conserving more energy. Most of the clustering protocols rotate the $\mathrm{CH}$ role so that the energy dissipation can be balanced in the network. However, regardless of the dynamic rotation of the $\mathrm{CH}$ role, the energy imbalance takes place due to communication distance between $\mathrm{SN}$ and the $\mathrm{CH}$. The location of the BS additionally influences the lifetime of the network as a longer distance will use more energy for communication. If the chosen $\mathrm{CHs}$ are nearer to the BS then it will dissipate less energy and if the CHs are at distant place then it will drastically deplete energy level. Some researchers have proposed multi-hop

*Madan Mohan Malviya University of Technology, Gorakhpur, India (anshucse.dwivedi@gmail.com) 
communication to the BS but it also depletes energy of $\mathrm{CH}$ nearer to the BS.

In this paper, we have propound a novel energy efficient fuzzy logic (NEEF) based clustering protocol for WSN that makes use of designed fuzzy system for energy efficacy while selecting the CHs thereby protracting network lifetime. The main contributions are highlighted as follows:

- The influential parameters that affect the battery level of SNs are identified from related work and segregated into primary and secondary factors.

- The primary factors considered are remnant energy level and communication cost to be borne by $\mathrm{CH}$, whereas secondary factors are the density of SN and its aloofnes to the BS.

- To emphasise the election of best suitable node for the $\mathrm{CH}$ role, weights are assigned to Fuzzy fitness output value (FF1 and FF2) after experimental evaluation through simulation.

- To balance the load of $\mathrm{CH}$, non- $\mathrm{CH}$ nodes choose their $\mathrm{CH}$ on the basis of chance obtained from designed fuzzy system which considers the load of $\mathrm{CH}$ node and distance from non- $\mathrm{CH}$ node to the considered $\mathrm{CH}$.

Subsequent part of this paper is organised as follows: Section 2 discusses literature survey. System model with network and energy dissipation model is discussed in section 3. A description about NEEF protocol is presented in section 4. Simulation experiment and result analysis is done in section 5 and section 6 provides concluding remarks. .

2. Relevant Work. This section discusses some pertinent clustering algorithms in WSN. Maximal clustering algorithms use rounds to describe the lifetime of WSN. Each round consists of $\mathrm{CH}$ selection, formation of cluster and the data collection. More the number of rounds, the longer will be the lifetime of WSN. LEACH [4] is a pioneering protocol in clustering algorithms. The goal of LEACH is to choose a node as CH in such a manner that every node gets an opportunity to become a $\mathrm{CH}$. The reason is that a $\mathrm{CH}$ node dissipates higher energy than non-CHs nodes, therefore, a node will not dissipate power by turning into the $\mathrm{CH}$ repeatedly. It uses randomness in selecting $\mathrm{CH}$, which may converge to no $\mathrm{CH}$ in a round. Gupta et al. implemented fuzzy logic for clustering of SN in WSN [12]. This work is an improvement over LEACH. The inputs for fuzzy systems are node degree, centrality and residual energy. However, it uses centralised approach by making use of BS for clustering. Centralised approaches are not easily scalable because of dependency on BS. The CH election mechanism relying on the Fuzzy reasoning (CHEF) was proposed by Kim et al. [13]. This protocol determines probability of node to act as $\mathrm{CH}$. It utilises the transmission range to the $\mathrm{BS}$ and the remnant node energy as fuzzy elements for the $\mathrm{CH}$ selection, unlike LEACH. The contrast between LEACH and CHEF shows more effective cluster formation in CHEF than on LEACH.

LEACH-FL is an enhanced LEACH variant with Fuzzy Logic [14]. It differs from LEACH in term of the factors used, viz. distance of node from the sink, type of battery used, and the density of a node. Selection of $\mathrm{CH}$ is a centralised process, similar to LEACH, handled by the BS, which computes the probability of a SN to become a CH. The authors of LEACH-FL have shown through experiments that the suggested protocol has reduced the energy dissipation rate. The lifetime of network using LEACH-FL protocol exceeds the lifespan of the network while considering LEACH. Lee and Chen have [15] propound a fuzzy logic-based clustering strategy in which a $\mathrm{CH}$ node is elected on basis of outstanding energy of a node and the expected outstanding energy. SEP-FL [16] is an enhanced variant of SEP [8], centred on the choice of CH by adjusting the remaining energy probabilities for each node. It offers a larger duration of stabilisation and a reduced duration of disturbance thereby improving node lifetime. The method is based on each node's distance from the BS and remnant energy level. EAUCF [17] is a fuzzy based unequal clustering approach. It proposes to lessen the energy depletion of $\mathrm{CHs}$ in pairs as they are either near to the BS or possibly have limited battery power left. EAUCF has a stronger output in terms of first node death (FND), quarter node death (QND) and relatively lower energy depletion in contrast to LEACH, CHEF [13] and EEUC [18].

MOFCA [19] is another technique of clustering in mobile sensor networks. Based on range to node and residual energy, $\mathrm{CHs}$ are determined. The radius of the $\mathrm{CH}$ is very important in relation to opportunity, which means that if a $\mathrm{CH}$ is closer to the BS and has more energy, it can gather and communicate more information. An enhanced variant of EAUCF [20] is FBUC [21] or Fuzzy Based Unequal Clustering. In addition, FBUC utilises a probabilistic limit function instead of a predefined limit number as compared to EAUCF [17] and provides a fuzzy input variable called degree of node which is used to select the $\mathrm{CH}$ during cluster radius contest. 
In different scenarios, FBUC exhibit lower power dissipation and longer network life than its counterpart in terms of first node death (FND) and last node death (LND). For a WSN to enhance lifetime, a CH selection scheme focused on fuzzy logic and particle swarm optimisation is suggested in [20]. DUCF [22] is another technique that uses fuzzy logic for clustering. Energy, proximity to the BS, and node temperature are regarded to be fuzzy inputs, and all nodes are selected in each round. This technique considers the most suitable node as a CH reducing energy consumption. FBECS [23], on the other hand, considers energy of SN, distance from the BS and density as an input to the FIS for selection of CH. It considers zonal network structure and corresponding probability during the clustering process. FBECS is capable of extending lifetime and stability time. DFLC [24] is another protocol based on the fuzzy logic that is carried out on nodes within the network in a distributed manner. DFLC considers network like a tree where it's nodes can be BS, cluster member and CH. DFLC is compared to ACAWT [25],LEACH, and CHEF [13]. Experimental results demonstrate that DFLC exhibit better performance than other algorithms in terms of chosen performance metrics. ECPF [26] also makes use of the fuzzy logic. Three procedures have been used to extend the life expectancy of the network. For fuzzy logic based calculation, ECPF utilises node degree and node centrality as fuzzy inputs for generating output for $\mathrm{CH}$ election.

SCHFTL [27], is based on fuzzy logic system, in which the sensor node uses different parameter at different levels. The first level parameters are remaining energy and centrality, the second level parameters used are communication quality and distance from the BS and the third level are total energy and DOS attack. With the help of this parameter, super cluster head is selected out of the chosen $\mathrm{CH}$. This protocol avoids the data overload, data loss and data retransmission, thereby increases the network life span. DFCR [28] routing protocol is propound that applies unequal clustering mechanism to solve the hotspot problem of WSN by minimising the size of cluster, that are closest to the BS. E-CAFL [29] is another routing protocol proposed to enhance CAFL [30] protocol by allowing node density. It uses three parameters, viz. distance from sink, remaining energy and density of node as input for FIS for estimation of rank for selecting the $\mathrm{CH}$.

In the above mentioned protocols, emphasis is not given to the parameters that majorly affect the energy level of SNs. We have determined the influential parameters and used them in fuzzy logic for best SNs selection in network. In most of the protocols mentioned in related work, they do not consider efficient cluster formation mechanism. We have fuzzy fitness value obtained during $\mathrm{CH}$ selection with other influential parameters while forming the clusters in our porposed work.

3. Preliminaries. This section presents the assumptions made in the network model for the proposed work in line with the energy dissipation model.

3.1. Network Model. Major presumptions that are made for the network are:

- SNs are randomly arranged in target area.

- After the deployment, all the nodes are stationary.

- BS doesn't have energy constraint.

- Deployed nodes are homogeneous in terms of resources.

- Every node has only one CH.

- The communication link is symmetric.

- The distance between two SN is determined by RSSI (Received Signal Strength Index).

- Initially, SN are unacquainted about their location.

3.2. Energy dissipation model. For the analysis of proposed work, the energy model adopted in [4] is employed. The energy of the network may be depleted in sensing, aggregation, amplification, transmission and reception. For transmitting and receiving $s$ bits over $d$ distance, energy dissipations are given by

$$
E_{T x}(s, d)= \begin{cases}s E_{\text {elec }}+s \epsilon_{f s} d^{2}, & d<d_{o} \\ s E_{\text {elec }}+s \epsilon_{m p} d^{4}, & d>d_{o}\end{cases}
$$

where do is a threshold which determines either free space or multipath model adopted and it can be calculated by $d_{o}=\sqrt{\epsilon_{f s} / \epsilon_{m p}}$.

$$
E_{R x}(s)=E_{R x-e l e c}(s)=s E_{\text {elec }}
$$




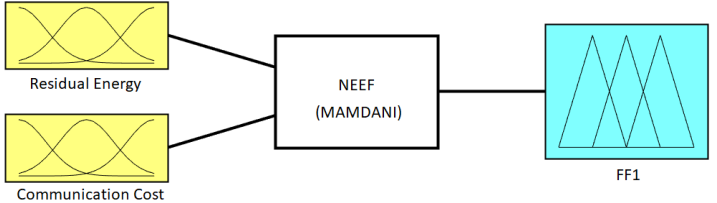

(a) FF1

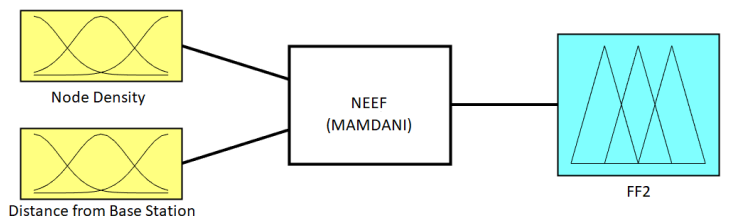

(b) FF2

FIG. 4.1. Designed FIS

In amplification of signal, energy dissipated is calculated by

$$
E_{a m p}= \begin{cases}\epsilon_{f s} d^{2}, & d<d_{o} \\ \epsilon_{m p} d^{4}, & d>d_{o}\end{cases}
$$

The communication cost to be borne by a $\mathrm{CH}$ in a round is calculated by

$$
E_{C H}=n s\left(E_{\text {elec }}+\epsilon_{f s} d_{B S}+E_{D A}\right)
$$

The total energy exhausted by a member of a cluster is calculated by

$$
E_{C M}=s\left(E_{\text {elec }}+\epsilon_{f s} d_{C H}\right)
$$

wherein $d_{C H}$ is the distance to its $\mathrm{CH}$.

4. Proposed NEEF protocol. The proposed NEEF protocol consists of four stages in a round i.e. pre-deployment stage, $\mathrm{CH}$ selection stage, cluster formation stage and data dissemination stage.

4.1. Pre-deployment stage. Before the deployment of SNs in the field, network administrator is required to allocate unique ID to the SNs. The information about the BS is also fed into the SN so that it can determine the BS during the operation of the network. For initial setup of the network and determining the neighbourhood, a TDMA slot is fed into each SN so that collision free broadcast can take place.

4.2. CH selelction stage. Once the deployment of $\mathrm{SN}$ is complete, it's time to select the optimal candidates to play the role of $\mathrm{CH}$. Since the role of $\mathrm{CH}$ is very crucial, fuzzy logic is applied to determine the optimal candidate. Fuzzy logic is mostly applied to solve the uncertainties in any system. For efficient selection of $\mathrm{CH}$ in WSN, there are several overlapping factors like remnant energy, distance between node to the BS, density in neighbourhood, communication cost, etc. Thus, Fuzzy logic is appropriate to solve the optimal CH selection problem as it can blend various factors dealing with uncertainties and provide better results. Since, the nodes are unaware about the location of the BS, a Hello_PKT(BSID) is broadcast by the BS so that every SN can estimate the aloofness from the BS. The SNs will make a broadcast as per the TDMA slot provided in pre-deployment phase. Once all the $\mathrm{SNs}$ are aware of the required parameters (distance to the BS, remnant energy, communication cost and density around node), the computation for $\mathrm{CH}$ candidature begins at each SN. Two Fuzzy inference system (FIS) have been designed for computing the Fuzzy fitness values (FF1 and FF2) of SNs as shown in Fig. 4.1.

For FF1, residual energy and communication cost are chosen as the input variables. The linguistic variables $(\mathrm{LV})$ chosen are Low $(\mathrm{Lw})$, Average $(\mathrm{Ag}), \operatorname{High}(\mathrm{Hg})$ and $\operatorname{Low}(\mathrm{Lw})$, Moderate( $\mathrm{Md})$, High( $\mathrm{Hg})$ respectively. LV for output variable are Very Weak (VW), Weak (W), Rather Weak (RW), Medium Weak (MW), Medium $(\mathrm{Mm})$, Rather Strong (RStr), Medium Strong (MStr), Strong (VStr) and Very Strong (VStr). The membership function (MF) for different LV which are derived for input and output variables are shown in Fig. 4.2. The FIS processes these input variables on the basis of LV and establishes a functional relationship between input and output LV on the basis of set of IF-THEN mapping rules. These rules which are used for calculating the FF1 is depicted in Table 4.1. These IF-THEN rules are evaluated using Mamdani inference method [31] which we have also depicted in Fig. 4.1 and Fig. 4.5. The reason for using this method is its simplicity and ability to easily interpret and draw conclusion on the basis of given IF-THEN rules. For defuzzification, we have used 


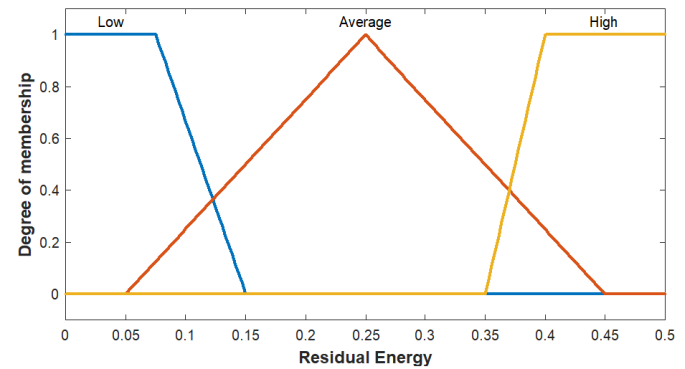

(a) Residual Energy

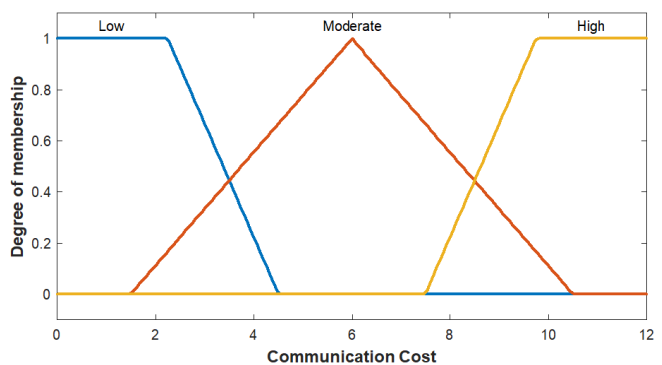

(b) Communication Cost

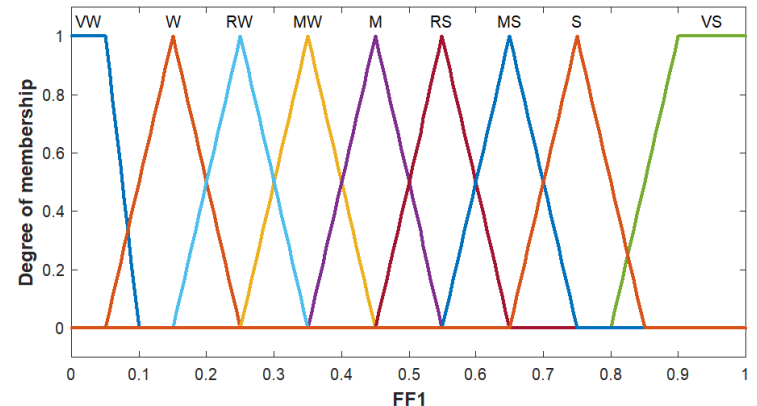

(c) Fuzzy Fitness Value (FF1)

FIG. 4.2. Membership Functions

TABle 4.1

Fuzzy Rules for FF1

\begin{tabular}{llll}
\hline Rule No. & Residual Energy & Communication cost & FF1 \\
\hline 1 & $\mathrm{Lw}$ & $\mathrm{Hg}$ & $\mathrm{VW}$ \\
2 & $\mathrm{Lw}$ & $\mathrm{Md}$ & $\mathrm{W}$ \\
3 & $\mathrm{Lw}$ & $\mathrm{Lw}$ & $\mathrm{RW}$ \\
4 & $\mathrm{Ag}$ & $\mathrm{Hg}$ & $\mathrm{MW}$ \\
5 & $\mathrm{Ag}$ & $\mathrm{Md}$ & $\mathrm{Mm}$ \\
6 & $\mathrm{Ag}$ & $\mathrm{Lw}$ & $\mathrm{RStr}$ \\
7 & $\mathrm{Hg}$ & $\mathrm{Hg}$ & $\mathrm{MStr}$ \\
8 & $\mathrm{Hg}$ & $\mathrm{Md}$ & $\mathrm{Str}$ \\
9 & $\mathrm{Hg}$ & $\mathrm{Lw}$ & VStr \\
\hline
\end{tabular}

COA method which is commonly used [30, 32]. For calculating the Fuzzy fitness value FF2, two parameters are considered: node density and distance from BS as shown in Fig. 4.1.

These two parameters have some significance during selection of $\mathrm{CH}$ candidate. Node density provides the estimation of neighbouring nodes which can reduce the intra-communication cost as more cluster members will lead to more dissipation of energy as well as coordination overhead. The objective is to distribute the load of $\mathrm{CH}$ role at par. Distance from BS determines the communication overhead which the $\mathrm{CH}$ has to borne for finally forwarding the data. If the number of neighbouring nodes is more and the BS is at distant place then more number of packets is to be forwarded to the BS which will deplete the energy of CH quickly. The LV for Node density is Scarce (Scr), Average (Ag) and Dense (Ds). Similarly, Near (Nr), Moderate (Md) and Distant (Dt) are the LV opted for Distance from the BS. For output variable FF2, the LV are Very Weak (VW), Weak(W), Medium Weak (MW), Medium(Mm), Strong (Str) and Very Strong(Str). Triangular and trapezoidal membership functions are chosen for interior values and boundary values respectively as shown in Fig. 4.3. The fuzzy IF-Then rules for mapping the input to output variables are depicted in Table 4.2. 


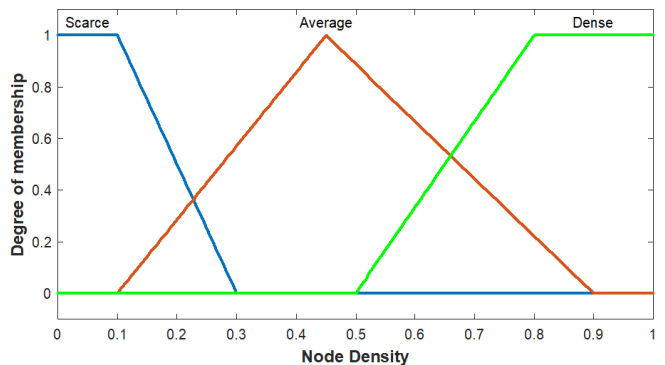

(a) Node density

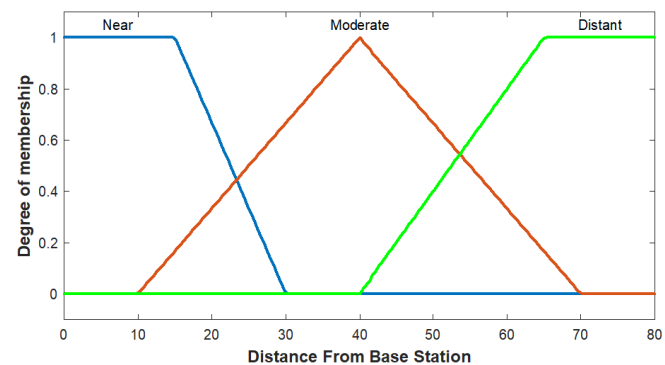

(b) Distance from $B S$

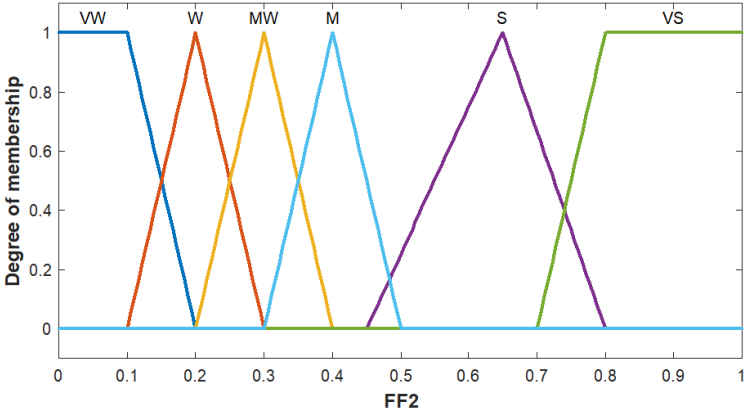

(c) Fuzzy Fitness Value (FF2)

Fig. 4.3. Membership Functions

TABLE 4.2

Fuzzy Rules for FF2

\begin{tabular}{llll}
\hline Rule No. & Node density & Distance from BS & FF2 \\
\hline 1 & Scr & Nr & MStr \\
2 & Scr & Md & MW \\
3 & Scr & Dt & MW \\
4 & $\mathrm{Ag}$ & $\mathrm{Nr}$ & Str \\
5 & $\mathrm{Ag}$ & $\mathrm{Md}$ & $\mathrm{Mm}$ \\
6 & $\mathrm{Ag}$ & $\mathrm{Dt}$ & $\mathrm{W}$ \\
7 & $\mathrm{Ds}$ & $\mathrm{Nr}$ & VStr \\
8 & $\mathrm{Ds}$ & $\mathrm{Md}$ & $\mathrm{Mtr}$ \\
9 & $\mathrm{Ds}$ & $\mathrm{Dt}$ & $\mathrm{VW}$ \\
\hline
\end{tabular}

After the computation of FF1 and FF2, every SN calculates its probability of being $\mathrm{CH}$ by

$$
S N(k) \cdot p r o b=\alpha \times S N(k) \cdot F F 1+\beta \times S N(k) . F F 2 \quad \text { s.t. }(\alpha+\beta)=1
$$

where $\alpha$ and $\beta$ are arbitrary constants.

We have considered the value of $\alpha$ and $\beta$ as 0.7 and 0.3 respectively as we got better results with these values as shown in Fig. 4.4. While carrying out experimental analysis of values assigned to $\alpha$ and $\beta$, we calculated the average $\mathrm{CH}$ to network energy ratio for $500^{\text {th }}$ round with varying values of $\alpha$ and $\beta$. The reason for weightage of $\alpha$ more than $\beta$ is that FF1 considers communication cost as well the remnant energy level of $\mathrm{SN}$ which are more influential in choosing efficient $\mathrm{CH}$. After each node computes the probabilistic value for its $\mathrm{CH}$ candidature, each node broadcast its probability. The $\mathrm{SNs}$ with highest probability are selected as $\mathrm{CH}$ candidates. Only $p \% \mathrm{CHs}$ are elected in each round. The process of selecting $\mathrm{CH}$ is defined in Algorithm 1. We have designed a FIS for computing chance of each $\mathrm{CH}$ as shown in Fig. 4.5.

There are three input variables: number of member nodes, communication distance to $\mathrm{CH}$ node and fuzzy fitness value $\mathrm{FF} 1$ of $\mathrm{CH}$ node. The output variable is Chance of $\mathrm{CH}$ which determines the probability of $\mathrm{CH}$ to be chosen by non-CH node. The LV for input and output variables are depicted in Table 4.3. Membership 


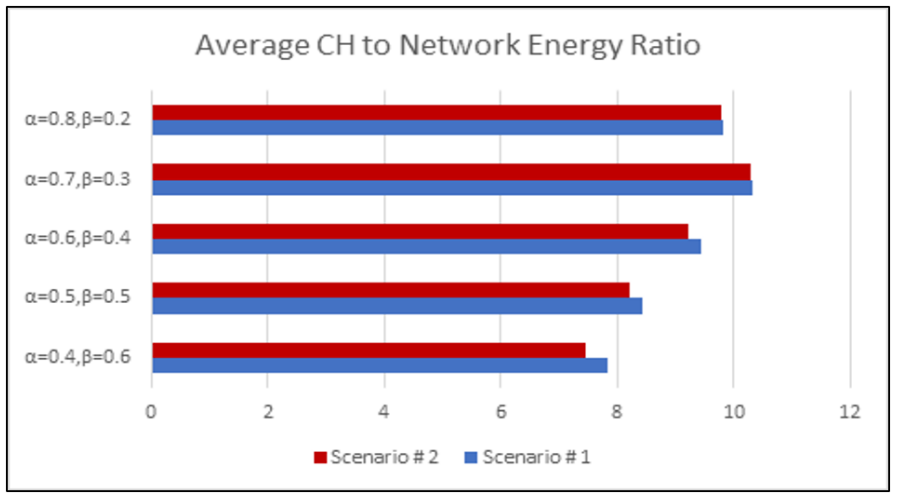

FIG. 4.4. Experimental evaluation of $\alpha$ and $\beta$ for average $C H$ to network energy ratio
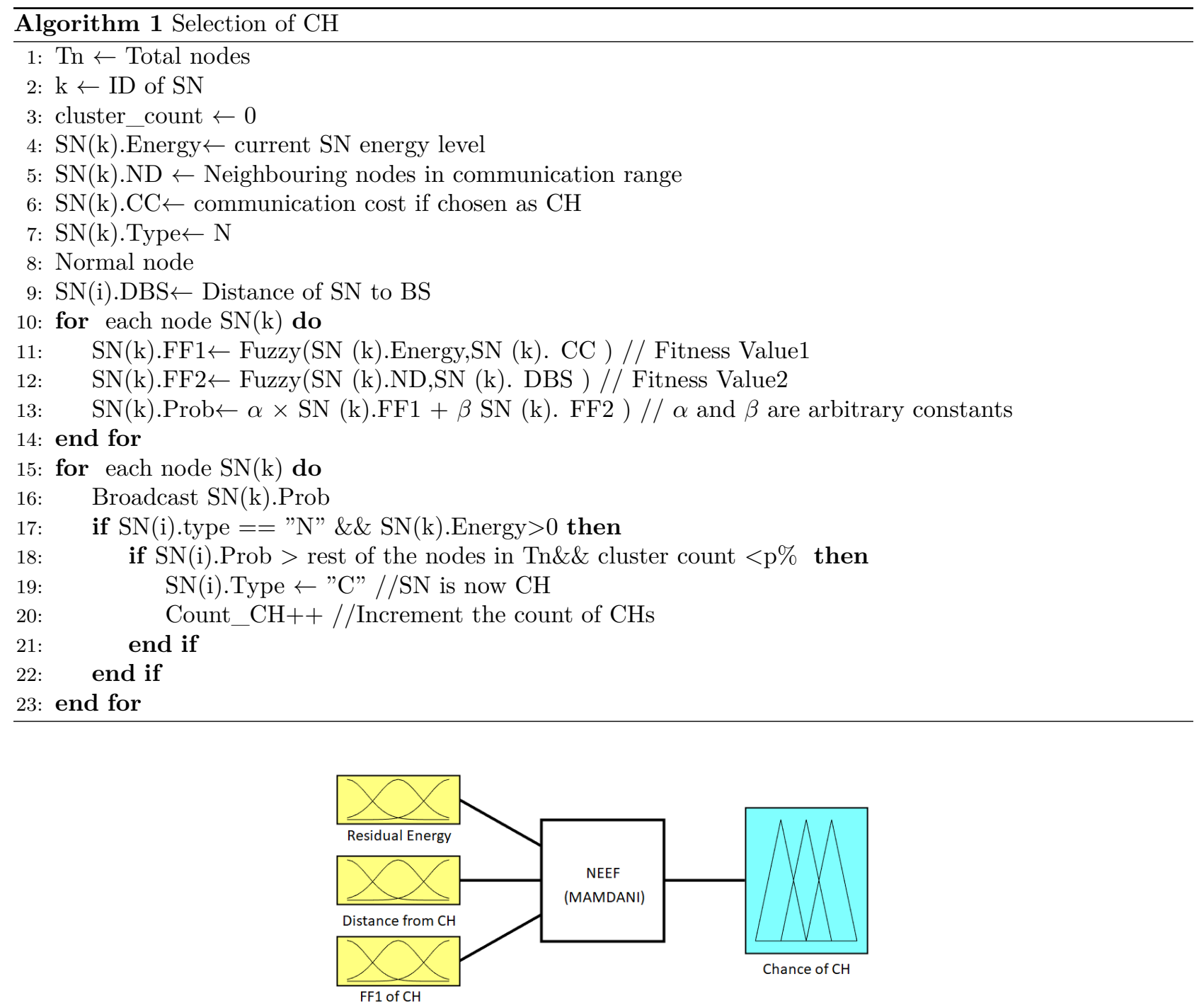

FIG. 4.5. FIS for computing Chance of $\mathrm{CH}$ 


\begin{tabular}{ll}
\hline Variable Name & Linguistic Variable \\
\hline Member nodes & Low(Lw),Medium(Mm),High(Hg) \\
Communication distance to CH node & Far(Fr),Medium(Mm),Near(Nr) \\
Fuzzy Fitness(FF) value 1 & Poor(Pr),Medium(Mm),High(Hg) \\
Chance of CH & Very Strong(VStr), Strong(Str), Medium Strong(MStr), \\
& Medium(Mm), Medium Weak(MW), Rather Weak(RW), \\
& Weak(W),Very Weak(VW) \\
\hline
\end{tabular}

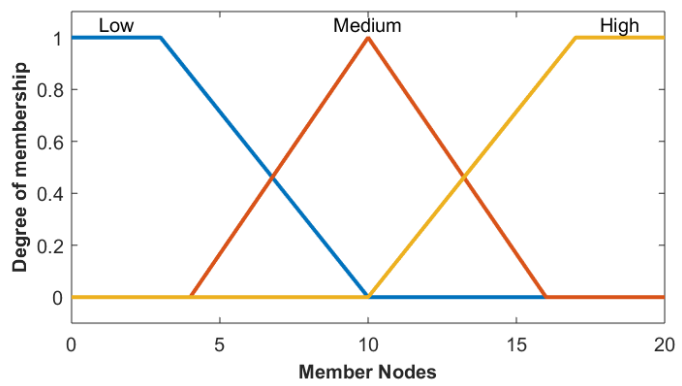

(a) Member nodes

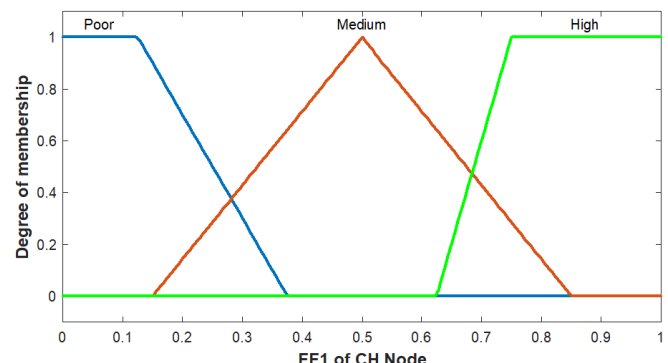

(c) Fuzzy Fitness Value (FF1)

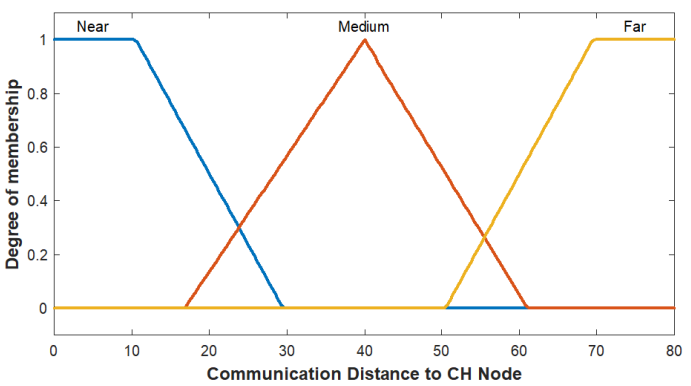

(b) Communication distance to $\mathrm{CH}$ Node

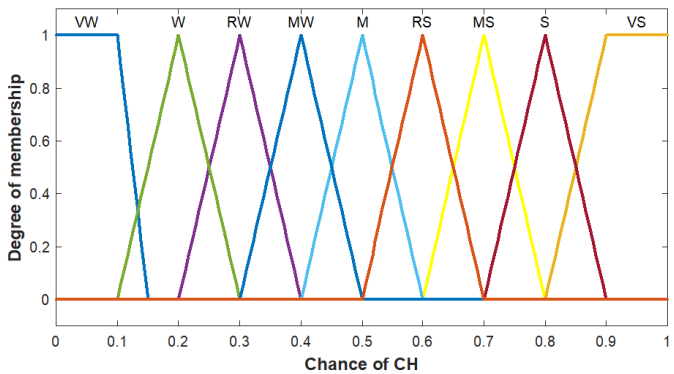

(d) Chance of $\mathrm{CH}$

FIG. 4.6. Membership Functions

functions of input and output variables are depicted in Fig. 4.6. IF-THEN rules for mapping input to output variables are depicted in Table 4.4. Each non- $\mathrm{CH}$ node computes the chance of each $\mathrm{CH}$ candidates and the $\mathrm{CH}$ candidate with maximum chance is chosen as optimum $\mathrm{CH}$ by the node. The non-CH node sends join request to the optimum $\mathrm{CH}$ node and receives acknowledgement from $\mathrm{CH}$ node with TDMA slot for data collection during the round. The cluster formation process is illustrated in Algorithm 2.

4.3. Data dissemination stage. Once the selection of $\mathrm{CHs}$ is completed and clusters are formed, a data dissemination stage comes into play. Data is generated from the target area on periodic basis. After sensing the target area, SN forwards the data to the $\mathrm{CH}$ as per the TDMA slot for collision free communication. Once the $\mathrm{CH}$ collects data from all the cluster members, it aggregates the data and transmits it to the BS for further processing. In this way, one round is concluded in NEEF protocol.

5. Simulation experiments and result analysis. NEEF is simulated and evaluated along with $\mathrm{SCH}-$ FTL [27] and DFCR [28] protocols using MATLAB. The field size is considered to be $100 \times 100 \mathrm{~m}^{2}$ with randomly scattered SNs. The operation of the network is split into rounds. For every round, $\mathrm{CH}$ selection, cluster formation and data dissemination take place. The parameters for simulation are described in Table 5.1which are kept similar to SCHFLT [27] and DFCR [28]. The performance metrics chosen for evaluation of proposed work are alive nodes per round, packets to the BS, average energy of network, stability period (FND), 
TABLE 4.4

Fuzzy rules for computing Chance of $\mathrm{CH}$

\begin{tabular}{|c|c|c|c|c|}
\hline Rule No. & $\mathrm{R}$ _Energy & DBS & Density & Rank \\
\hline 1 & Lw & $\mathrm{Fr}$ & $\operatorname{Pr}$ & VW \\
\hline 2 & Lw & $\mathrm{Fr}$ & $\mathrm{Mm}$ & $\mathrm{Vw}$ \\
\hline 3 & Lw & $\mathrm{Fr}$ & $\mathrm{Hg}$ & $\mathrm{W}$ \\
\hline 4 & Lw & $\mathrm{Mm}$ & $\operatorname{Pr}$ & W \\
\hline 5 & Lw & $\mathrm{Mm}$ & $\mathrm{Mm}$ & $\mathrm{W}$ \\
\hline 6 & Lw & $\mathrm{Mm}$ & $\mathrm{Hg}$ & RW \\
\hline 7 & Lw & $\mathrm{Nr}$ & $\operatorname{Pr}$ & RW \\
\hline 8 & Lw & $\mathrm{Nr}$ & $\mathrm{Mm}$ & MW \\
\hline 9 & $\mathrm{Mm}$ & $\mathrm{Nr}$ & $\mathrm{Hg}$ & MW \\
\hline 10 & $\mathrm{Mm}$ & $\mathrm{Fr}$ & $\operatorname{Pr}$ & RW \\
\hline 11 & $\mathrm{Mm}$ & $\mathrm{Fr}$ & $\mathrm{Mm}$ & RW \\
\hline 12 & $\mathrm{Mm}$ & $\mathrm{Fr}$ & $\mathrm{Hg}$ & MW \\
\hline 13 & $\mathrm{Mm}$ & $\mathrm{Mm}$ & $\mathrm{Pr}$ & RW \\
\hline 14 & $\mathrm{Mm}$ & $\mathrm{Mm}$ & $\mathrm{Mm}$ & MW \\
\hline 15 & $\mathrm{Mm}$ & $\mathrm{Mm}$ & $\mathrm{Hg}$ & MW \\
\hline 16 & $\mathrm{Mm}$ & $\mathrm{Nr}$ & $\mathrm{Pr}$ & MW \\
\hline 17 & $\mathrm{Hg}$ & $\mathrm{Nr}$ & $\mathrm{Mm}$ & $\mathrm{Mm}$ \\
\hline 18 & $\mathrm{Hg}$ & $\mathrm{Nr}$ & $\mathrm{Hg}$ & RStr \\
\hline 19 & $\mathrm{Hg}$ & Fr & $\operatorname{Pr}$ & $\mathrm{Mw}$ \\
\hline 20 & $\mathrm{Hg}$ & $\mathrm{Fr}$ & $\mathrm{Mm}$ & $\mathrm{Mm}$ \\
\hline 21 & $\mathrm{Hg}$ & Fr & $\mathrm{Hg}$ & RStr \\
\hline 22 & $\mathrm{Hg}$ & $\mathrm{Mm}$ & $\operatorname{Pr}$ & RStr \\
\hline 23 & $\mathrm{Hg}$ & $\mathrm{Mm}$ & $\mathrm{Mm}$ & MStr \\
\hline 24 & $\mathrm{Hg}$ & $\mathrm{Mm}$ & $\mathrm{Hg}$ & Str \\
\hline 25 & $\mathrm{Hg}$ & $\mathrm{Nr}$ & $\operatorname{Pr}$ & MStr \\
\hline 26 & $\mathrm{Hg}$ & $\mathrm{Nr}$ & $\mathrm{Mm}$ & Str \\
\hline 27 & $\mathrm{Hg}$ & $\mathrm{Nr}$ & $\mathrm{Hg}$ & Vstr \\
\hline
\end{tabular}

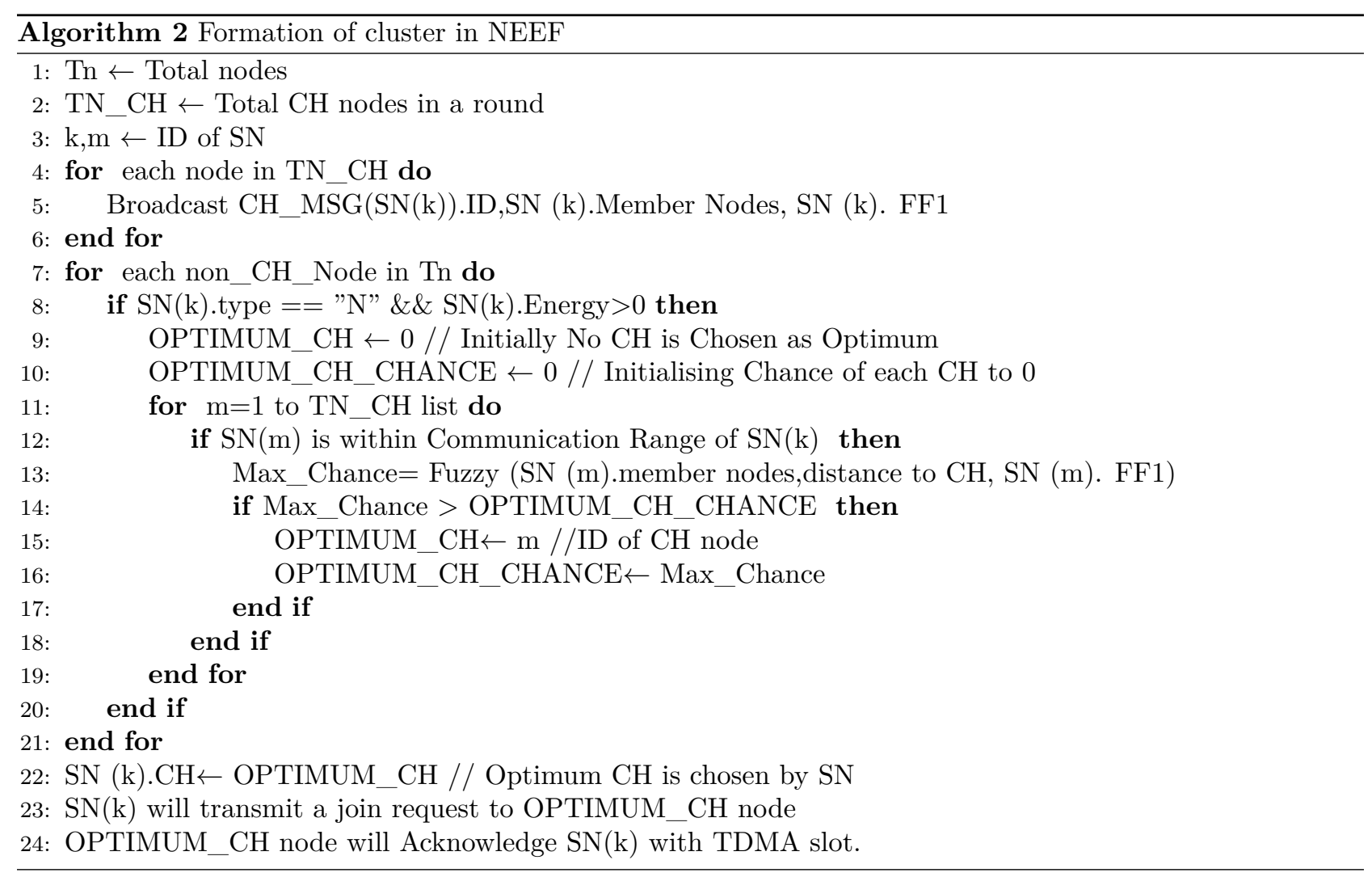




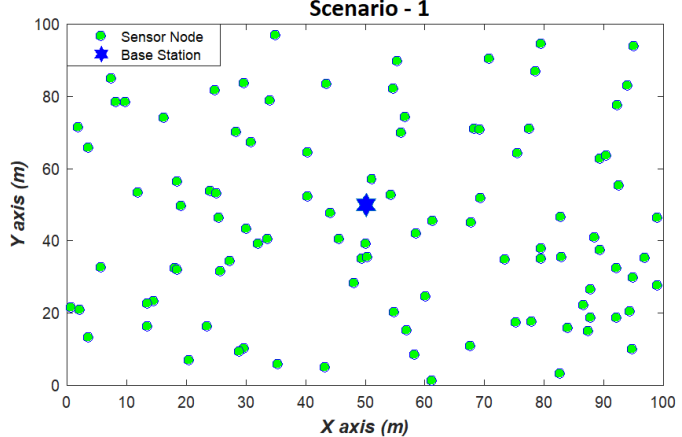

(a) Network Structure for S\#1

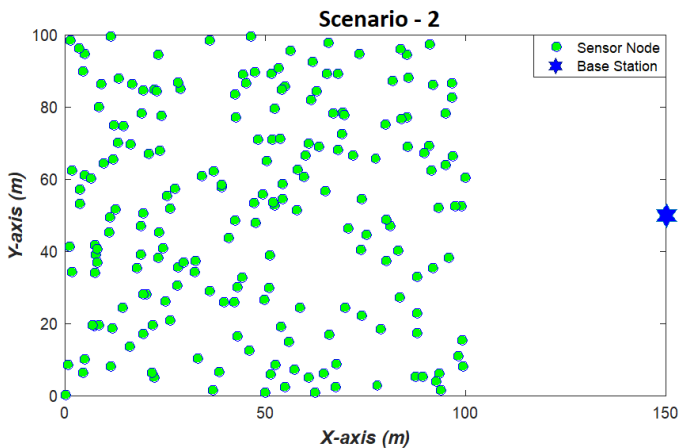

(b) Network Structure for S\#2

FIG. 5.1. Network structure.

TABLE 5.1

Simulation Parameters and their values

\begin{tabular}{lll}
\hline Parameters & Symbol & Values \\
\hline Total SNs in Field & $\mathrm{N}$ & 100,200 \\
Amplifier energy for free space & $\epsilon_{f s}$ & $10 \mathrm{pJ} / \mathrm{bit} / \mathrm{m}^{2}$ \\
BS location & $\mathrm{BS}$ & $(50,50),(150,50)$ \\
Amplifier energy for multipath & $\epsilon_{m p}$ & $0.0013 \mathrm{pJ} / \mathrm{bit} / \mathrm{m}^{4}$ \\
Energy of SN before deployment & $E_{o}$ & $0.5 \mathrm{~J} / 1.0 \mathrm{~J}$ \\
Data packet Size & $\mathrm{M}$ & $4000 \mathrm{bits}$ \\
Data Fusion & $E_{D A}$ & $5 \mathrm{~nJ} / \mathrm{bit} / \mathrm{report}$ \\
Arbitrary constants & $\alpha, \beta$ & $0.7,0.3$ \\
Percentage of CH probability & $p \%$ & 10 \\
Electronic Circuitry & $E_{\text {elec }}$ & $50 \mathrm{~nJ} / \mathrm{bit}$ \\
\hline
\end{tabular}

QND, HND, average energy of chosen CHs. These metrics will evaluate the protocol from every perspective conforming the enhancement in lifetime of the network. We have carried out simulation more than 50 times and the results were normalised. The graphs depicted are instance of one of the simulations carried out so that clear picture about the performance of all the simulated fuzzy based protocols can be perceived.

5.1. Network structure. In conducting simulation experiments, we have considered two network structure/scenarios as depicted in Fig. 5.1. In scenario $1(\mathrm{~S} \# 1)$, the BS is positioned at the centre of the field and scenario 2 ( $\mathrm{S} \# 2$ ) considers the BS located at far off place from the field. The reason for choosing two scenarios is that this protocol can satisfy all the applications of WSN where the BS is either within the vicinity or beyond the vicinity.

5.2. Alive nodes. With the focus on longer lifetime with maximum coverage, alive nodes have huge impact on WSN. More the number of alive nodes, longer will be the lifetime of the network. Fig. 5.2 depicts the number of alive SNs in the field after each rounds for both the scenarios. It can be clearly witnessed that NEEF performs better than SCHFTL and DFCR protocol as it has more alive nodes after each round as equated to SCHFTL and DFCR for both the scenarios. In scenario 1, for up to 1500 rounds, almost all the nodes are dead for SCHFTL and DFCR protocols whereas more than $90 \%$ nodes are alive in case of NEEF protocol. For scenario 2, for up to 1500 rounds, more than $90 \%$ nodes are alive in the network for NEEF protocol whereas no node is alive for SCHFTL protocol and more than $95 \%$ nodes are dead in case of DFCR protocol. Obtained result from Fig.5.2(a,b) clearly unveil the balanced load distribution among the deployed SNs.

5.3. Throughput. Collecting information from the target area is the ultimate objective of WSN. Successful delivery of more information to the BS reveals better design of protocol. Fig. 5.3 exhibits the number of successful packet delivery to the BS during span of the network. We can see that that NEEF protocol 


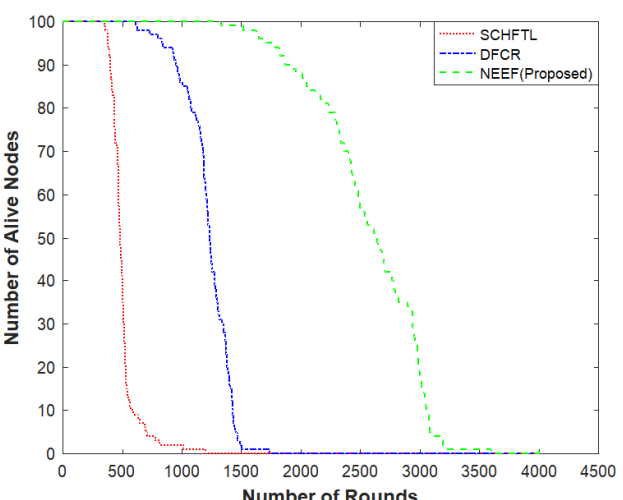

(a) $S \# 1$

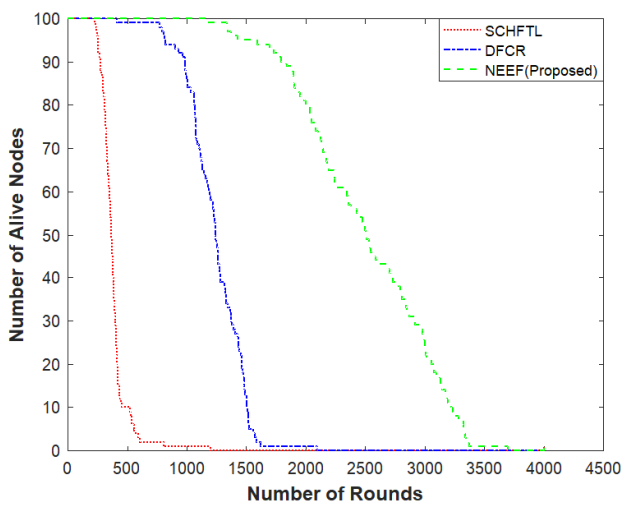

(b) $S \# 2$

FIG. 5.2. Alive nodes w.r.t rounds

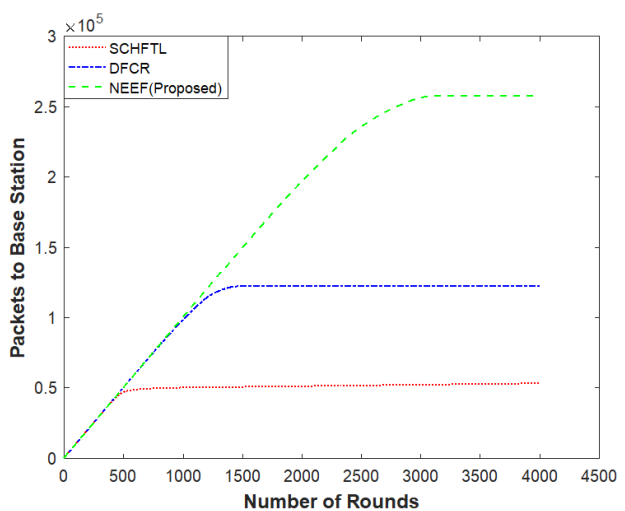

(a) $S \# 1$

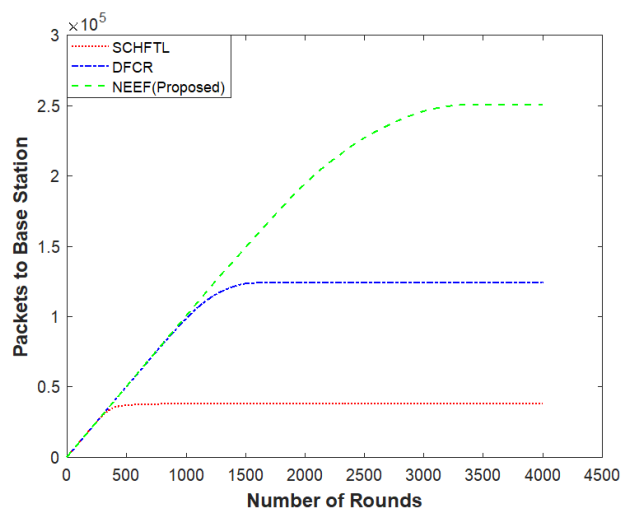

(b) $S \# 2$

FIG. 5.3. Packets to the BS w.r.t. rounds

has forwarded more packets to the BS as compared to SCHFTL and DFCR protocol. Since, more number of nodes are alive per round in NEEF protocol, therefore, more information is forwared to the BS. Every WSN is expected to forward as much information as possible with longer lifetime and this purpose is served by NEEF protocol.

5.4. Average energy of network. The dissipation rate of energy of SN may affect the lifetime of the network. If the network is dissipating energy quickly then the lifetime will decrease resulting in incomplete coverage of target area. Fig. 5.4 discuss about average energy of network per round. It can be seen that average energy of NEEF protocol for each round is much more than SCHFTL and DFCR protocol for both the scenarios. The reason behind the better performance is the consideration of crucial parameters like communcation cost to be borne by SN if chosen as $\mathrm{CH}$ and its remnant energy level during the selection of CH. This balances the load resulting in more average energy of the network. Stability period or First node dead determines the reliability of the network [8]. If a protocol exhibit better stability period then it's clear that it has more reliability as it ensure the complete coverage of the network because all the $\mathrm{SN}$ are alive in the network till that stability period.

5.5. First Node Dead,Quarter Node Dead and Half Node Dead. FND ensures that the network is reliable as the deployed nodes are covering the target area intactly. QND and HND are checkpoints which determine the rate at which the nodes are expiring. Fig. 5.5 shows the rounds in which the simulated protocols have FND, QND and HND. The reason why we have not considered Last Node Dead (LND) is that after the 


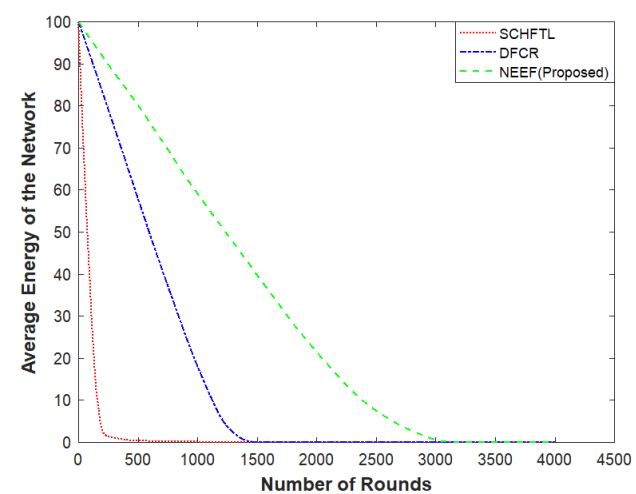

(a) $S \# 1$

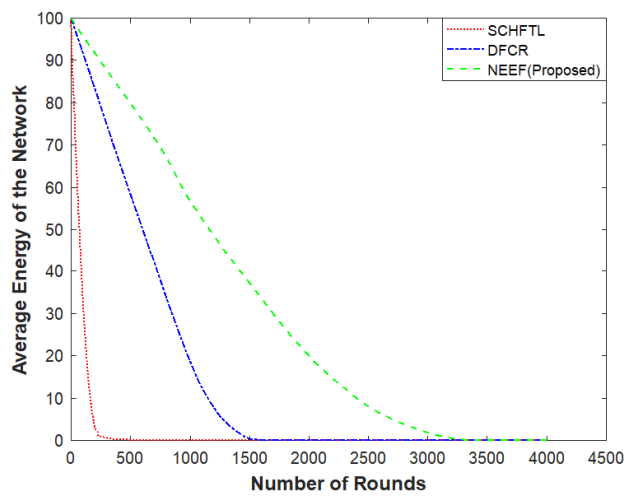

(b) $S \# 2$

Fig. 5.4. Average energy $(J)$ per round

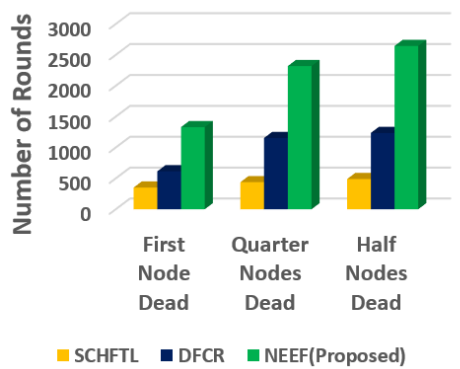

(a) $S \# 1$

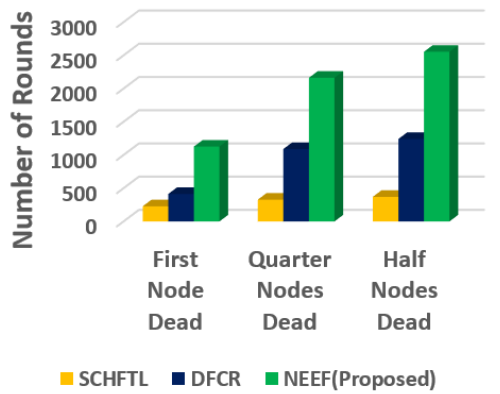

(b) $S \# 2$

FIG. 5.5. First Node Dead, Quarter Node Dead and Half Node Dead

death of $50 \%$ node, there is no reliability about the coverage of the target field. From experimental analysis, we have seen that some protocols continue up to 1000 rounds more with their last node which has no relevance as single node is incapable of covering all the target field. For FND, NEEF has improved over SCHFTL and DFCR by $278.63 \%$ and $116.09 \%$ for scenario 1 . In case of scenario 2, the improvement of FND for NEEF is further increased having 398.85\% and $172.81 \%$ over SCHFTL and DFCR respectively. If we talk about QND, NEEF has shown increment of $429.22 \%$ and $100.86 \%$ over SCHFTL and DFCR respectively for scenario 1 and $561.16 \%$ and $98.34 \%$ over SCHFTL and DFCR respectively for scenario 2. For Half Node Death, in case of Scenario 1, the obtained results are $440.69 \%$ and $114.08 \%$ better than SCHFTL and DFCR respectively and for Scenario 2, the improvements are $590 \%$ and $105.72 \%$ over SCHFTL and DFCR respectively.

5.6. Average energy of $\mathbf{C H}$. This performance metric ensures that the nodes which are having better energy levels along with other primary and secondary factors are turned into $\mathrm{CH}$. In Fig. 5.6, we have chosen average energy of $\mathrm{CHs}$ in lifetime of the network as one of metric because it depicts how energy bundled SNs are chosen to take the challenging role of $\mathrm{CH}$. It is witnessed that the average energy of $\mathrm{CH}$ for scenario 1 is improved by $986.34 \%$ and $59.25 \%$ as compared to SCHFTL and DFCR protocol. For scenario 2, NEEF performs $1104.91 \%$ and $67.93 \%$ better than SCHFTL and DFCR protocol. The cause of the poor performance of SCHFTL is that it has chosen some random values for the parameters like DOS, communication quality and total delay which does not give deep insight of the SN capability while selecting the $\mathrm{CH}$ candidate. In case of DFCR protocol, it considers the remnant energy and aloofness from the BS as crucial factors for election of $\mathrm{CH}$ where intra cluster communication cost is neglected which results in poor performance as compared to NEEF protocol. 


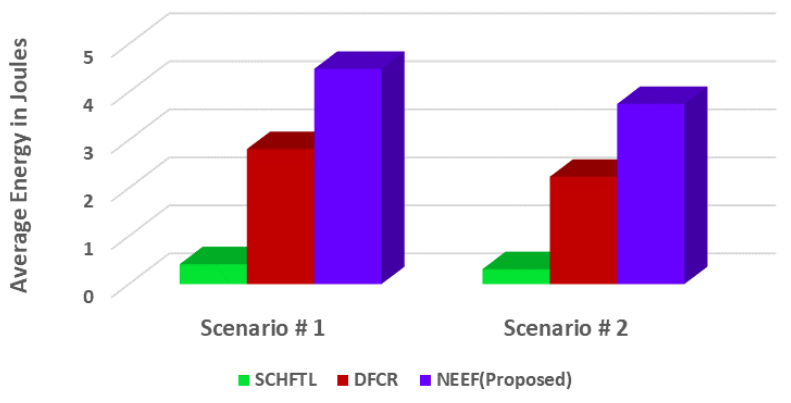

FIG. 5.6. Average Energy of $\mathrm{CH}$ for both scenarios

6. Conclusion. NEEF is proposed for resource constrained WSN. This protocol has considered two fuzzy fitness values obtained from primary and secondary factors fed to designed Fuzzy Inference System. Primary factors include remnant energy and communication cost whereas secondary factors considered are node density and distance to the base station. With experimental observations, primary and secondary factors are used in appropriate proportion for selection of best cluster head candidates. For balancing the cluster head role, noncluster head members use fuzzy logic for choosing their cluster heads. NEEF exhibits tremendous improvement over SCHFTL and DFCR protocol in terms of protracted lifetime, stability period, and better average energy of chosen cluster heads and communication of more information to the base station. This protocol is suitable for applications where either the base station can be put at the centre or beyond the target field. In future, we will perform simulation experiments on mobile sensor nodes.

\section{REFERENCES}

[1] P.Rawat, K. Singh, H.Chaouchi, and J.M.Bonnin. Wireless sensor networks: a survey on recent developments and potential synergies. The Journal of Supercomputing, 68(1):1-48, Apr 2014.

[2] M. Mehdi Afsar and Mohammad H. Tayarani-N. Clustering in sensor networks: A literature survey. Journal of Network and Computer Applications, 46:198-226, Nov 2014.

[3] P.S. Mehra, M.N. Doja, And B. Alam. Enhanced stable period for two level and multilevel heterogeneous model for distant base station in wireless sensor network, volume 379, page 751-759. 2016.

[4] W.R. Heinzelman, A. Chandrakasan, and H. Balakrishnan. Energy-efficient communication protocol for wireless microsensor networks. In Proceedings of the 33rd Annual Hawaii International Conference on System Sciences, volume vol.1, page 10. IEEE Comput. Soc, 2000.

[5] P. S. Mehra, M. N. Doja, and B. Alam. Stability Enhancement in LEACH (SE-LEACH) for Homogeneous WSN. EAI Endorsed Transactions on Scalable Information Systems, 6(20):e5, 2019.

[6] S. Lindsey and C.S. Raghavendra. Pegasis: Power-efficient gathering in sensor information systems. In Proceedings, IEEE Aerospace Conference, volume 3, pages 3-1125-3-1130. IEEE, 2002.

[7] O.Younis AND S.FAhmy. Heed: a hybrid, energy-efficient, distributed clustering approach for ad hoc sensor networks. Mobile Computing, IEEE Transactions on, 3(4):366-379, 2004.

[8] G. Smaragdakis, I. Matta, and a. Bestavros. SEP: A stable election protocol for clustered heterogeneous wireless sensor networks. Second International Workshop on Sensor and Actor Network Protocols and Applications (SANPA 2004), 2004.

[9] P. S. Mehra, M. N. Doja, And B. Alam. Low energy adaptive stable energy efficient (LEASE) protocol for wireless sensor network. In 2015 1st International Conference on Futuristic Trends in Computational Analysis and Knowledge Management, ABLAZE 2015, page 484-488, 2015.

[10] H.Zhou, Y.Wu, Y.Hu, And G.XIE. A novel stable selection and reliable transmission protocol for clustered heterogeneous wireless sensor networks. Computer Communications, 33(15):1843-1849, Sep 2010.

[11] P.S. Mehra, M.N. Doja, And B. Alam. Stable Period Enhancement for Zonal (SPEZ)-Based Clustering in Heterogeneous WSN, volume 79, page 887-896. Springer, Singapore, 2018.

[12] I. Gupta, D. Riordan, and S. Sampaldi. Cluster-head election using fuzzy logic for wireless sensor networks. In $3 \mathrm{rd}$ Annual Communication Networks and Services Research Conference (CNSR'05), page 255-260. IEEE, 2005.

[13] J Kim, S PARK, Y HAn, AND T Chung. Chef: Cluster head election mechanism using fuzzy logic in wireless sensor networks. In Proceedings of 10th International Conference on Advanced Communication Technology, pages 654-659, 2008.

[14] G.RAN, H.ZHANG, AND S.Gong. Improving on leach protocol of wireless sensor networks using fuzzy logic. Journal of Information \& Computational Science, 7(3):767-775, 2010. 
[15] J.S.Lee And W.L.Cheng. Fuzzy-logic-based clustering approach for wireless sensor networks using energy predication. IEEE Sensors Journal, 12(9):2891-2897, Sep 2012.

[16] F.Zhang, Q.Y.Zhang, AND Z.M.Sun. Ict2tsk: An improved clustering algorithm for wsn using a type-2 takagi-sugeno-kang fuzzy logic system. In 2013 IEEE Symposium on Wireless Technology \& Applications ISWTA), page 153-158. IEEE, Sep 2013.

[17] B.Mostafa, C.SaAd, and H.Abderrahmane. Fuzzy logic approach to improving stable election protocol for clustered heterogeneous wireless sensor networks. Journal of Theoretical and Applied Information Technology, 53(3):334-339, 2013.

[18] C.Li, M.Ye, G.Chen, ANd J.Wu. An energy-efficient unequal clustering mechanism for wireless sensor networks. In $2 n d$ IEEE International Conference on Mobile Ad-hoc and Sensor Systems, MASS 2005, volume 2005, page 597-604, 2005.

[19] S.A.Sert, H.Bagci, And A.Yazici. Mofca: Multi-objective fuzzy clustering algorithm for wireless sensor networks. Applied Soft Computing, 30:151-165, May 2015.

[20] Z.M.Zahedi, R.Akbari, M.Shokouhifar, F.Safaei, and A.Jalali. Swarm intelligence based fuzzy routing protocol for clustered wireless sensor networks. Expert Systems with Applications, 55:313-328, Aug 2016.

[21] R. Logambigai and A. Kannan. Fuzzy logic based unequal clustering for wireless sensor networks. Wireless Networks, 22(3):945-957, Apr 2016.

[22] B. Baranidharan and B. Santhi. Ducf: Distributed load balancing unequal clustering in wireless sensor networks using fuzzy approach. Applied Soft Computing, 40:495-506, Mar 2016.

[23] P. S. Mehra, M. N. Doja, and B. Alam. Fuzzy based enhanced cluster head selection (FBECS) for wsn. Journal of King Saud University - Science, Apr 2018.

[24] D.R.D.ADHIKARY AND D.K.MALLICK. An energy aware fuzzy approach to unequal clustering in wireless sensor networks, applied soft computing, 13(4), pp. 1741-1749, 2013. Journal of ICT Research and Applications, 11(1):56-77, Apr 2017.

[25] A.Alaybeyoglu and Aysegul. A distributed fuzzy logic-based root selection algorithm for wireless sensor networks. Computers 83 Electrical Engineering, 41(C):216-225, Jan 2015.

[26] Q.WAng, D.Lin, P.YAng, and Z.Zhang. A fuzzy-logic based energy-efficient clustering algorithm for the wireless sensor networks. In 2018 26th International Conference on Software, Telecommunications and Computer Networks (SoftCOM), page 1-6. IEEE, Sep 2018.

[27] M.Ayati, M.H.Ghayyoumi, And A.K.Mohammadiyan. A fuzzy three-level clustering method for lifetime improvement of wireless sensor networks. Annales des Telecommunications/Annals of Telecommunications, 73(7-8):535-546, 2018.

[28] N.Mazumdar And H.OM. Distributed fuzzy approach to unequal clustering and routing algorithm for wireless sensor networks. International Journal of Communication Systems, 31(12):e3709, Aug 2018.

[29] P. S. Mehra, M.N. Doja, and B.Alam. Enhanced clustering algorithm based on fuzzy logic (E-CAFL) for wsn. Scalable Computing: Practice and Experience, 20(1):41-54, Mar 2019.

[30] H. El Alami and A. Najid, Fuzzy Logic Based Clustering Algorithm for Wireless Sensor Networks, International Journal of Fuzzy System Applications (IJFSA), 6 (2017), pp. 63-82.

[31] E.H.Mamdani. Application of fuzzy logic to approximate reasoning using linguistic synthesis. IEEE Transactions on Computers, C-26(12):1182-1191, Dec 1977.

[32] P.Nayak and A.Devulapalli. A fuzzy logic-based clustering algorithm for wsn to extend the network lifetime. IEEE Sensors Journal, 16(1):137-144, Jan 2016.

Edited by: Dana Petcu

Received: Apr 28, 2020

Accepted: Jul 6, 2020 